\title{
A study on the relationship of type III radio bursts CME and solar flares during the active period October-November 2003
}

\author{
Michaella Thanassa ${ }^{1}$, Eleftheria Mitsakou ${ }^{1}$, Panagiota \\ Preka-Papadema $^{1}$, Xenophon Moussas ${ }^{1}$, Panagiotis Tsitsipis ${ }^{2}$ \\ and Athanasios Kontogeorgos ${ }^{2}$ \\ ${ }^{1}$ Department of Physics, University of Athens, 15784 Athens, Greece \\ ${ }^{2}$ Department of Electronics, Technological Education Institute of Lamia, Lamia, Greece
}

\begin{abstract}
Within a period of intense activity (20 October to 5 November 2003), the injection and propagation of near relativistic electrons, resulted in hundreds of type III bursts recorded by the ARTEMISIV radio spectrograph $(20-650 \mathrm{MHz})$. For a number of these type III events association with GOES SXR/H $\alpha$ flare and/or SOHO/LASCO CME was established. We study the variation of characteristic type III parameters and their relationship with features of the associated flares and/or CMEs.
\end{abstract}

Keywords. Sun: coronal mass ejections (CMEs), Sun: flares,Sun: radio radiation

\section{Introduction}

In the period 20 October-5 November 2003 a global complex consisting of three large, remote but connected active regions: AR 0484 (Complex), AR 0486 (Complex) and AR 0488 (Bipolar) produced an abundance of solar energetic phenomena (intense flares, fast CMEs). Several type III bursts were also recorded.

The importance of the study is two fold: Firstly, the electron beams exciting the type III radio emission, stream along the coronal magnetic lines, thus tracing magnetic structures; secondly the origin of the same beams is directly associated with particle acceleration mechanisms in the lower corona.

In this report we expand on the results of a previous study (Mitsakou et al. 2006) examining the relationship between the metric solar type III radio bursts and coronal mass ejections (CMEs), and soft X-ray (SXR) flares.

\section{Data selection and analysis}

Our data set consists of 124 metric type III bursts and groups recorded by the ARTEMISIV radio spectrograph (Caroubalos et al. 2001, 2006, also Kontogeorgos et al. 2006a, $2006 \mathrm{~b}$ ) in the range $20-650 \mathrm{MHz}$ within the active period 20 October-5 November 2003; those were associated with flares ( $\mathrm{H} \alpha$ and GOES SXR) from the Solar Geophysical Data (SGD) reports and CMEs from the SOHO/LASCO lists; the association of bursts with GOES/SXR enhancements or flares was established within a time window of about 5 min. From our sample we have eliminated the periods of extreme activity accompanied by type II/IV burst as it was impossible to isolate and analyse the type III bursts in this case. 

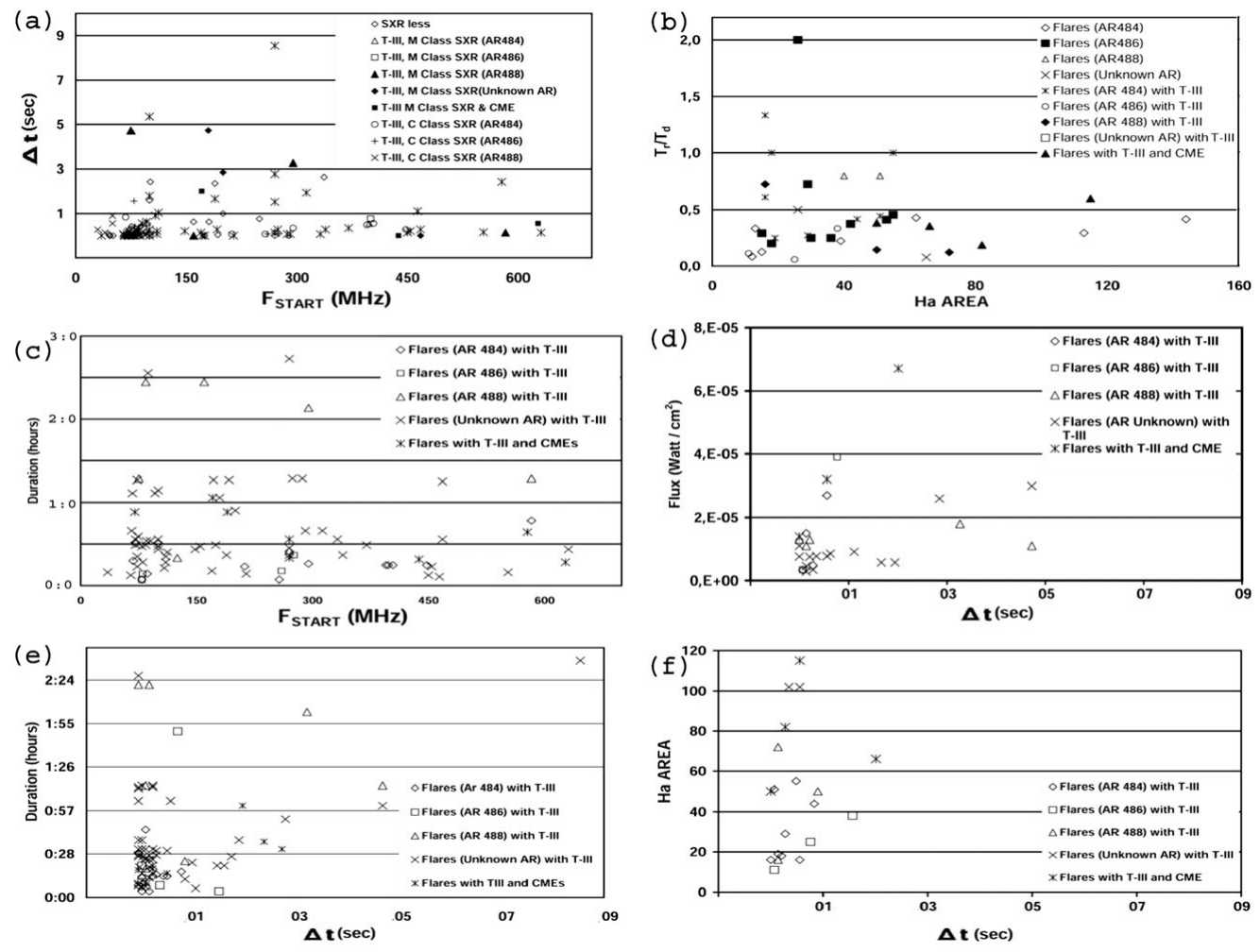

Figure 1. (a) Type III duration $\Delta t$ as function of start frequency $F_{S T A R T}$, (b) Ratio of SXR rise to decay time $\left(t_{r} / t_{d}\right)$ as a function of $\mathrm{H} \alpha$ AREA, (c) SXR Duration (D) as a function of start frequency $F_{S T A R T}$, (d) SXR FLUX as a function of Type III duration $\Delta t$, (e) SXR Duration (D) as a function of Type III duration $\Delta t$, (f) $\mathrm{H} \alpha$ AREA as a function of Type III duration $\Delta t$.

From the data sample we deduce a $69 \%$ association of type III bursts with recorded flares or SXR enhancements; $9 \%$ are also spatially and temporally associated with fast and small-width CME (jet-CMEs). A significant fraction (31\%) were detected between successive SXR flux maxima (they were labeled as SXRless in the text) though not always in the same active region as the SXR flare. The lack of SXR enhancement in SXRless type III bursts was probably the result of increased SXR background which prevented detection. As regards event characteristics we have examined:

- The type III starting frequency $\left(F_{S T A R T}\right)$ depends on the depth of the exciter acceleration in the solar corona; it is, usually, less than $300 \mathrm{MHz}\left(F_{S T A R T}<300 \mathrm{MHz}\right)$ with the SXRless events having $F_{S T A R T}<150 \mathrm{MHz}$. The majority of the jet-CME associated bursts have $F_{S T A R T}>200 \mathrm{MHz}$. In AR 0484 we have occasionally $F_{S T A R T} \approx 500 \mathrm{MHz}$ (cf. figure 1a).

- The type III (or group) duration $(\Delta t)$ is characteristic of the group size. We have found that $\Delta t$ depends on $F_{S T A R T}$ in a rather complicated way: For $F_{S T A R T}>350 \mathrm{MHz}$ we have recorded only isolated bursts (Low $\Delta t$ ), for $F_{S T A R T}<350 \mathrm{MHz}$, both groups and isolated bursts appear (cf. figure 1a).

- SXR Flux and H $\alpha$ flare Area : as we have excluded from our sample the type II/IV periods, most of the Type III-only associated flares are of class $\mathrm{C}$ and small ( $\mathrm{H} \alpha$ area $<60$ and $\mathrm{H} \alpha$ area $<40$ in the case of AR 0486). Only the jet-CMEs associated flares appear to have $\mathrm{H} \alpha$ area $>50$ (cf. figure $1 \mathrm{~b}$ ). 
- SXR duration (D), rise time $\left(t_{r}\right)$, and decay time $\left(t_{d}\right)$ : Mostly they have $t_{r} / t_{d}<1 / 2$ (cf. figure $1 \mathrm{~b} \& 1 \mathrm{c}$ ). The flare SXR duration (D) ranges between a few minutes and up to 2 hours, with the majority at approximately 30 minutes. The jet-CME-associated flares and the majority of the flares in AR 0488 have durations in excess of 30 minutes.

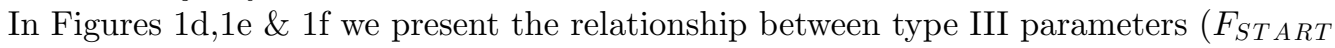
and $\Delta t$ ) with the associated flare characteristics (H $\alpha$ AREA, SXR Flux and Duration).

\section{Final Remarks}

The type III bursts and groups, in the absence of type II/IV activity, are, in general associated with medium to small flares since electron acceleration to $\approx 0.3 c$ does not require much energy. The start frequency, in the majority of events, is less than $300 \mathrm{MHz}$ with some exceptions of isolated bursts.

The appearance of type III bursts and groups is favored by an increase in SXR flux and $\mathrm{H} \alpha$ Area. Increasing SXR duration (D) on the other hand, does not favor the appearance of bursts of the type III family. In fact as D increases, in the bipolar AR 0488 in particular, the starting frequency and duration of the type IIIs decrease; we note that AR 0488 gives many (50\%) LDEs as opposed to the other two ARs.

The two complex active regions (AR 0484 and AR 0486) produced mostly a large number of short duration type IIIs, from the bipolar AR $048850 \%$ of type IIIs had duration $(\Delta T)$ exceeding 2 sec.

\section{References}

Caroubalos, C., Alissandrakis, C. E., Hillaris, A., Preka-Papadema, P., Polygiannakis, J., Moussas, X., Tsitsipis, P., Kontogeorgos, A., Petoussis, V., Bouratzis, C., Bougeret, J. L., Dumas, G., \& Nindos, A., N. Solomos, ed., Recent Advances in Astronomy and Astrophysics, vol. 848 of American Institute of Physics Conference Series 2006, pages 864-873

Caroubalos, C., Maroulis, D., Patavalis, N., Bougeret, J. L., Dumas, G., Perche, C., Alissandrakis, C., Hillaris, A., Moussas, X., Preka-Papadema, P., Kontogeorgos, A., Tsitsipis, P., \& Kanelakis, G. 2001, Experimental Astronomy, 11, 23

Kontogeorgos, A., Tsitsipis, P., Caroubalos, C., Moussas, X., Preka-Papadema, P., Hilaris, A., Petoussis, V., Bouratzis, C., Bougeret, J. L., Alissandrakis, C. E., \& Dumas, G. 2006a, Experimental Astronomy, 21, 41

Kontogeorgos, A., Tsitsipis, P., Moussas, X., Preka-Papadema, G., Hillaris, A., Caroubalos, C., Alissandrakis, C., Bougeret, J. L., \& Dumas, G. 2006b, Space Sci. Rev., 122, 169

Mitsakou, E., Thanasa, M., Preka-Papadema, P., Moussas, X., Hillaris, A., Caroubalos, C., Alissandrakis, C. E., Tsitsipis, P., Kontogeorgos, A., Bougeret, J. L., \& Dumas, G., N. Solomos, ed., Recent Advances in Astronomy and Astrophysics, vol. 848 of American Institute of Physics Conference Series, 2006, pages 234-237 